\title{
A Nonsense Mutation in the Carboxyl-terminal Domain of Type X Collagen Causes Haploinsufficiency in Schmid Metaphyseal Chondrodysplasia
}

\author{
Danny Chan, ${ }^{*}$ Yi Ma Weng, ${ }^{\star}$ H. Kerr Graham, ${ }^{\ddagger}$ David O. Sillence, ${ }^{\S}$ and John F. Bateman ${ }^{\star}$ \\ *Orthopedic Molecular Biology Research Unit, Department of Pediatrics, University of Melbourne, Parkville, Melbourne, Australia 3052; \\ ${ }^{\ddagger}$ Department of Orthopedic Surgery, Royal Children’s Hospital, Parkville, Melbourne, Australia 3052; and ${ }^{\S}$ Department of Pediatrics, The \\ New Children's Hospital, Parramatta, Sydney, Australia 2124
}

\begin{abstract}
Type X collagen is a short-chain homotrimeric collagen expressed in the hypertrophic zone of calcifying cartilage. The clustering of mutations in the carboxyl-terminal NC1 domain in Schmid metaphyseal chondrodysplasia (SMCD) suggested a critical role for this type $X$ collagen domain, but since no direct analysis of cartilage has been conducted in SMCD patients, the mechanisms of type $X$ collagen dysfunction remain controversial. To resolve this problem, we obtained SMCD growth plate cartilage, determined the type $X$ collagen mutation, and analyzed the expression of mutant and normal type $X$ collagen mRNA and protein. The mutation was a single nucleotide substitution that changed the $\mathrm{Tyr}^{632}$ codon (TAC) to a stop codon (TAA). However, analysis of the expression of the normal and mutant allele transcripts in growth plate cartilage by reverse transcription PCR, restriction enzyme mapping, and a single nucleotide primer extension assay, demonstrated that only normal mRNA was present. The lack of mutant mRNA is most likely the result of nonsense-mediated mRNA decay, a common fate for transcripts carrying premature termination mutations. Furthermore, no mutant protein was detected by immunoblotting cartilage extracts. Our data indicates that a functionally null allele leading to type $X$ collagen haploinsufficiency is the molecular basis of SMCD in this patient. (J. Clin. Invest. 1998. 101:1490-1499.) Key words: bone development - cartilage - COL10A1 - osteochondrodysplasia • mutation
\end{abstract}

\section{Introduction}

Schmid metaphyseal chondrodysplasia (SMCD; MIM 156500)1 is a relatively mild autosomal dominant disorder of the osseous skeleton resulting from growth plate cartilage abnormalities. The SMCD phenotype shows clinical variability, but is characterized by short stature, coxa vara, and genu varum leading to a waddling gait. Radiological findings include flar-

Address correspondence to John F. Bateman, Ph.D., Department of Pediatrics, University of Melbourne, Royal Children's Hospital, Parkville, Victoria 3052, Australia. FAX: +61-3-9345-6367; E-mail: bateman@cryptic.rch.unimelb.edu.au

1. Abbreviations used in this paper: RT, reverse transcription; SMCD, Schmid metaphyseal chondrodysplasia; wt, wild type.

J. Clin. Invest.

(C) The American Society for Clinical Investigation, Inc. 0021-9738/98/04/1490/10 \$2.00

Volume 101, Number 7, April 1998, 1490-1499

http://www.jci.org ing of the metaphysis and a wide irregular growth plate, especially at the knees (1).

SMCD results from mutations in the gene for type $X$ collagen, COL10A1 (2-4). Type X collagen is a short-chain collagen with a restricted pattern of expression confined to the terminally differentiated hypertrophic chondrocytes of growth plate cartilage during endochondral bone growth (5-8), fracture repair (9), and as a consequence of joint degeneration in osteoarthritis (10). The type $\mathrm{X}$ collagen molecule is a homotrimer of three $\alpha 1(\mathrm{X})$ chains encoded by a condensed gene (COL10A1) of three exons, one of which (exon 3) codes for the majority of the polypeptide chain including the entire triple helical domain $(11,12)$. The human $\alpha 1(\mathrm{X})$ homotrimer consists of three distinct protein domains. The short triple helical domain, COL1 (amino acids 57-519), containing eight imperfections in the Gly-X-Y triplet repeat sequence, is flanked by a small nonhelical globular NC2 domain at the amino terminus (amino acids 19-56) and a larger more conserved nonhelical carboxyl-terminal NC1 domain (amino acids 520-680; 11, 13).

Over 25 mutations of COL10A1, recently summarized by Wallis et al. (4), have been characterized in SMCD covering the gamut of amino acid substitutions, nonsense mutations, and deletions resulting in predicted protein truncation. The clustering of these mutations within the highly conserved NC1 suggests a critical role for this domain in type $\mathrm{X}$ collagen synthesis, molecular assembly, and function. In one of these patients, in vitro expression of mutant $\alpha 1$ (X)G618V cDNA demonstrated that the mutation compromised NC1 assembly (3). Further site-directed mutagenesis and in vitro expression and assembly studies demonstrated that chains with several other SMCD NC1 mutations were unable to assemble into homotrimers. Expression in transiently transfected cells further confirmed that the mutant chains were unable to form stable homotrimeric molecules, and that secretion was also severely impaired (14). Furthermore, in in vitro coexpression and assembly experiments, mutant chains containing NC1 truncations (14) were unable to associate with normal chains or to interfere with normal chain assembly. However, the trace amounts of heterotrimer assembly detected with some $\alpha 1(\mathrm{X})$ NC1 missense mutants suggested that while in general functional haploinsufficiency is the major defect in SMCD, it is possible that some $\mathrm{NC1}$ mutations may allow mutant/normal heterotrimer assembly, contributing to the clinical phenotype.

Initial studies on mice homozygous for a Colloal knockout-produced null allele appeared normal (15), suggesting that type $\mathrm{X}$ collagen depletion caused no gross phenotypic changes in mice. In contrast, subsequent independent detailed analysis of an analogous type $\mathrm{X}$ collagen-deficient mouse demonstrated phenotypic changes that at least in part, resembled SMCD (16). Transgenic mice expressing a chicken $\alpha 1(X)$ chain with an internal helical deletion have severe skeletal abnormalities (17) more akin to human spondylometaphyseal 
dysplasia. While type $\mathrm{X}$ collagen in the growth plate of this transgenic model could be depleted as a result of degradation of mutant/normal hybrid type X collagen trimers, it is likely that the severe phenotype resulted from a dominant-negative effect of the mutant-containing molecules on matrix assembly and organization.

Thus the genotype/phenotype relationship of type X collagen mutations in human SMCD and the mouse models, and the corollary of that, the physiological role of type X collagen in the growth plate, remains an important unresolved question. To determine if type $\mathrm{X}$ collagen haploinsufficiency or a dominant-negative effect of mutant allele expression is the molecular basis of human SMCD, it is clearly critical to analyze biochemically the type $\mathrm{X}$ collagen in growth plate cartilage samples from SMCD patients. In this study, we characterize a novel heterozygous type $\mathrm{X}$ collagen premature termination mutation in the NC1 domain (Y632X). In direct analysis of growth plate cartilage tissue, we demonstrate that only the normal allele was expressed. The inability to detect mutant mRNA or mutant type $X$ collagen protein indicates that in this patient, a functionally null $\alpha \mathbf{1}(\mathrm{X})$ allele leading to haploinsufficiency is the molecular basis causing SMCD.

\section{Methods}

Clinical description. An 11-year-old girl presented with a waddling gait for evaluation. The main clinical features were short stature, lordosis of the lumbar spine, anterior pelvic tilt, and asymmetric deformities of the knees with genu varum on the right and genu valgum on the left with bilateral coxa vara and a very high riding great trochanter (Fig. 1). X rays also confirmed lumbar lordosis but no scoliosis and the chest $\mathrm{x}$ ray was largely normal. The condition was diagnosed as the SCMD. Cartilage samples were obtained during orthopedic procedures with parental consent and approval of the Institutional Ethics Committee.

Amplification of COL10A1 genomic sequences. Genomic DNA was extracted from whole blood (18), and the coding sequence was PCR-amplified using sets of overlapping oligonucleotide primers previously described to produce four PCR products of $287 \mathrm{bp}$ from exon 2, and 850,983 , and 745 bp from exon 3 (3). The polymerase chain re- actions were carried out using the DNA amplification kit from Perkin Elmer Cetus (Foster City, CA). The cycling conditions were $94^{\circ} \mathrm{C}$ for $1.5 \mathrm{~min}, 62^{\circ} \mathrm{C}$ for $1.5 \mathrm{~min}$, and $72^{\circ} \mathrm{C}$ for $2 \mathrm{~min}$ for 30 cycles. The amplified fragments were purified by gel electrophoresis on $2.5 \%$ (wt/vol) NuSieve agarose (FMC Bioproducts, Rockland, ME) and recovered using Geneclean (Bio 101, Inc., Vista, CA).

Formation of heteroduplexes and chemical cleavage. The PCR conditions were adjusted to favor heteroduplex formation in reamplification of purified PCR products using limiting primer concentration. Each set of primers for the PCR was serially diluted to determine the optimal primer concentration, defined by the dilution at which the yield of the product is limited by the concentration of primers after 20 cycles using $5 \mathrm{ng}$ of purified PCR products as template. In general, the optimal concentration is about $25 \mathrm{ng}$ of each primer per reaction. In some reactions, $2.5 \mathrm{ng}$ of purified PCR products from a normal individual and the patient were combined before reamplification. However, this combination did not result in any increase in the amount of heteroduplexes formed. The cycling conditions were $94^{\circ} \mathrm{C}$ for 1.5 min, $62^{\circ} \mathrm{C}$ for $1.5 \mathrm{~min}$, and $72^{\circ} \mathrm{C}$ for $2 \mathrm{~min}$ for 20 cycles. The amplified heteroduplex fragments were purified by gel electrophoresis on $2.5 \%$ (wt/vol) NuSieve agarose and recovered using Geneclean. Before chemical cleavage to detect mispaired bases (19), $\sim 200 \mathrm{ng}$ of the purified heteroduplex DNA was radioactively labeled at the $5^{\prime}$ ends with $\left[\gamma^{-32} \mathrm{P}\right]$ ATP $(3,000 \mathrm{Ci} / \mathrm{mmole}, 10 \mathrm{mCi} / \mathrm{ml}$; NEN Life Science Products (Boston, MA) by polynucleotide kinase.

Cloning and sequencing of PCR products. A 510-bp fragment containing the mismatch was generated using primers BX1, 5'CAGGGGGTAACAGGAATGCC-3' (bp 1726-1745), and HX6 5'CTTTTCAGCCTACCTCCATA-3' (bp 2235-2216), purified by agarose electrophoresis, recovered using Geneclean, kinased, and cloned into the Sma I site of pBluscript II SK (+) (Stratagene Inc., La Jolla, CA). Positive clones were selected and isolated plasmids were sequenced using a cycle-sequencing kit from Perkin Elmer Cetus Instruments (Emeryville, CA).

Restriction mapping and single nucleotide primer extension analysis. Total RNA was extracted from $8-\mu \mathrm{m}$ transverse frozen sections from the patient and control growth plate cartilage as previously described (20). The entire coding region of the type X collagen was amplified by reverse transcription RT (PCR) (Perkin Elmer Cetus) from cDNA produced using oligo-dT as primer for reverse transcription. The conditions for cDNA synthesis were those recommended by the manufacturer. Primers HX11, GAGAATATGCTGCCAATACCCT (bp 92-115) and HX6 were used in the PCR step to amplify a 2.1-kb

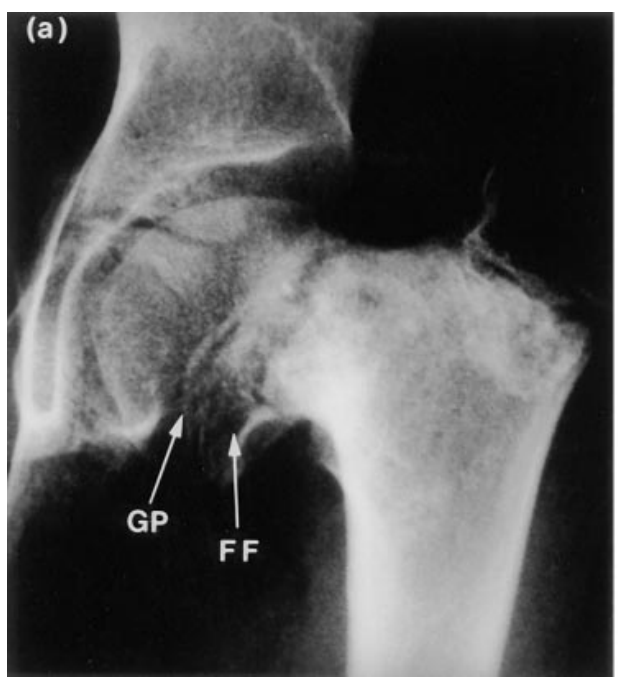

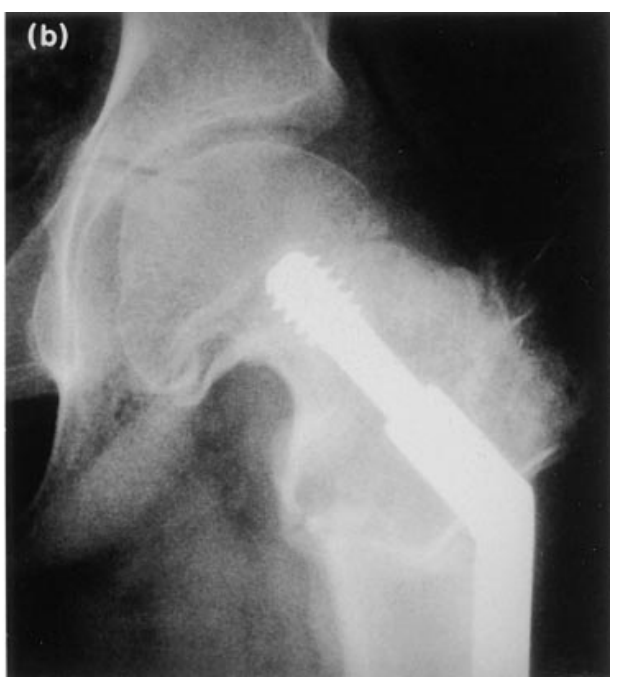

Figure 1. Pre- and post-operative $\mathrm{x}$ rays. (a) Anteroposterior $\mathrm{x}$ ray of the left hip showing the growth plate $(G P)$, a pathological fatigue fracture $(F F)$ of the femoral neck, coxa vara, and a very high riding great trochanter. The tip of the trochanter is well above the top of the femoral head. The neck/shaft angle is $80^{\circ}$. (b) The postoperative $\mathrm{x}$ ray shows that the coxa vera has been corrected by an intertrochanteric valgus femoral osteotomy. The neck shaft angle is now $130^{\circ}$. The fatigue fracture has healed, and the tip of the great trochanter is now below the height of the femoral head. The metallic fixation device crosses both the fatigue fracture and the growth plate. During its insertion, a sample of the growth plate cartilage was removed for subsequent analysis. 
fragment, and the conditions were $95^{\circ} \mathrm{C}$ for $5 \mathrm{~min}, 62^{\circ} \mathrm{C}$ for $1.5 \mathrm{~min}$, and $72^{\circ} \mathrm{C}$ for $3 \mathrm{~min}$ for one cycle, and then $95^{\circ} \mathrm{C}$ for $1.5 \mathrm{~min}, 62^{\circ} \mathrm{C}$ for $1.5 \mathrm{~min}$, and $72^{\circ} \mathrm{C}$ for $2 \mathrm{~min}$ over 30 cycles, and finally $7 \mathrm{~min}$ at $72^{\circ} \mathrm{C}$. The 510-bp fragment (bp 1726-2235) encompassing the mutation was amplified from the 2.1-kb RT-PCR fragment using primers BX1 and HX6. As the mutation introduced a new Bsr I cleavage site, this fragment was amplified from the patient and a control, digested with Bsr I. The resultant products were analyzed by electrophoresis on a $2.5 \%$ agarose gel stained with ethidium bromide. Single nucleotide extension analysis was also used to estimate the concentration of normal and mutant message. Approximately $5 \mathrm{ng}$ of the $2.1-\mathrm{kb}$ cDNA fragment was used as a template for single nucleotide extension with an oligonucleotide (5'-TGAATACACCAAAGGCTA-3', bp 19741991) that primes one base $5^{\prime}$ to the mutation. The reaction was carried out at $95^{\circ} \mathrm{C}$ for $1 \mathrm{~min}, 42^{\circ} \mathrm{C}$ for $2 \mathrm{~min}$, and $72^{\circ} \mathrm{C}$ for $1 \mathrm{~min}$ in $10 \mu \mathrm{l}$ of $10 \mathrm{mM}$ Tris/ $\mathrm{HCl}, \mathrm{pH} 8.3$, containing $50 \mathrm{mM} \mathrm{KCl,} 2 \mathrm{mM} \mathrm{MgCl}_{2}$, $0.01 \%(\mathrm{wt} / \mathrm{vol})$ gelatin, $1 \mu \mathrm{M}$ primer, and $2 \mu \mathrm{Ci}$ of either $\left[\alpha{ }^{32} \mathrm{P}\right] \mathrm{dCTP}$ or $\left[\alpha-{ }^{32} \mathrm{P}\right] \mathrm{dATP}$ (NEN Life Science Products) in the absence of nonradioactive dNTPs. The products were analyzed on a $15 \%(\mathrm{wt} / \mathrm{vol})$ denaturing polyacrylamide gel containing $7 \mathrm{M}$ urea and the radioactivity of the extended products were quantified using a phosphorimager (Molecular Dynamics, Sunnyvale, CA).

In vitro expression of type $X$ collagen constructs. A 365-bp fragment containing the mutation was released by digestion with Nsi I and Pfl MI from the plasmid isolated from a positive clone after sequencing. This fragment was introduced into a full-length human type $\mathrm{X}$ cDNA clone pTM1-h10wt to produce a mutant construct, pTM1Y632X, according to procedures described previously (14). The plasmids were translated using the TNT T7 polymerase-coupled transcription and translation system (Promega Corp., Madison, WI) as previously described in a reaction volume of $12.5 \mu \mathrm{l}$ in the presence of canine microsomal membranes (Promega Corp.). The translated products were labeled with translation-grade $\mathrm{L}-\left[{ }^{35} \mathrm{~S}\right]$ methionine (1,000 Ci/mmol, NEN Life Science Products) and analyzed by SDS/ PAGE $(3,14)$.

Type $X$ collagen analysis from growth plate cartilage. Femoral neck growth plate cartilage from the patient and femoral growth plate cartilage from a normal $20 \mathrm{wk}$ fetus were extracted in parallel. Intact type $\mathrm{X}$ collagen was extracted from $8-\mu \mathrm{m}$ transverse frozen section of growth plate cartilage with a $50 \mathrm{mM}$ Tris/ $\mathrm{HCl}$ buffer, $\mathrm{pH} 7.5$, containing $1 \mathrm{M} \mathrm{NaCl}, 10 \mathrm{mM}$ DTT, $5 \mathrm{mM}$ EDTA, $0.1 \mathrm{mM}$ phenylmethysulfonyl fluoride, and $10 \mathrm{mM} \mathrm{N}$-ethylmaleimide for $24 \mathrm{~h}$ at $4^{\circ} \mathrm{C}$. Total proteins in the extracts were precipitated with $10 \%(\mathrm{wt} / \mathrm{vol})$ trichloroacetic acid and resolved on a 7.5\% SDS/polyacrylamide gel (3). Before electrophoresis, the samples were denatured at $100^{\circ} \mathrm{C}$ for $10 \mathrm{~min}$. Type $\mathrm{X}$ collagen chains were detected by immunoblot analysis using an antibody specific for the triple helical domain (gift from Dr. Gary Gibson, Henry Ford Hospital, Detroit, MI), previously demonstrated to react with wild-type chains as well as chains containing NC1 mutations (14). Alkaline phosphatase-conjugated protein-G (Bio-Rad Laboratories, Hercules, CA) was used to detect bound antibodies with a BM chemiluminescence substrate from Boehringer Mannheim. Portions of growth-plate-containing cartilage were also cultured as described by Gibson et al. (21) to produce $\left[{ }^{35} \mathrm{~S}\right]$ methionine-labeled type $\mathrm{X}$ collagen. Radioactively labeled type $\mathrm{X}$ collagen secreted into the medium was recovered by immunoprecipitation with another type $\mathrm{X}$ collagen antibody specific for the carboxyl-terminal NC1 domain (gift from Dr. Olena Jacenko, University of Pennsylvania Veterinary School, Philadelphia, PA) and analyzed on a $7.5 \%$ SDS/polyacrylamide gel.

SDS-PAGE. Type X collagen chains were resolved on $7.5 \%(\mathrm{wt} /$ vol) polyacrylamide separating gels with a $3.5 \%$ (wt/vol) stacking gel. Before electrophoresis, samples were diluted with loading buffer to give a final concentration of $0.125 \mathrm{mM}$ Tris $/ \mathrm{HCl}, \mathrm{pH} 6.8$, containing $2 \%$ (wt/vol) SDS, $2 \mathrm{M}$ urea, and $10 \mathrm{mM} \mathrm{DTT}$, and denatured at $60^{\circ} \mathrm{C}$ for $10 \mathrm{~min}$ or otherwise as indicated. The approximate molecular size of the in vitro expression products were determined by comparison to Rainbow molecular weight standards (Amersham Life Science, Buck- inghamshire, United Kingdom). Electrophoresis conditions and fluorography of radioactive gels have been described previously $(22,23)$.

Immunohistology and ultrastructural analysis. For histological analysis, longitudinal paraffin sections $(5 \mu \mathrm{m})$ along the femoral growth plate cartilage were dewaxed and stained with hematoxylin and eosin. Sections for immunohistology were rehydrated in PBS after dewaxing and treatment with decreasing graded ethanol. To expose the collagenous matrix determinants, sections were treated with testicular hyaluronidase (Sigma Chemical Co., St. Louis, MO) for 30 min at $37^{\circ} \mathrm{C}$, blocked with $10 \%(\mathrm{vol} / \mathrm{vol})$ horse serum in PBS before hybridization overnight at $4{ }^{\circ} \mathrm{C}$ with either an antibody to type $\mathrm{X}$ collagen $\mathrm{NC1}$ domain or an antibody specific for the amino telopeptide of the $\alpha 2$ (I) chain of type I collagen. Sections were washed with PBS containing $0.05 \%$ (wt/vol) Tween-20, and bound antibodies were detected with a second antibody, donkey anti-rabbit IgG, conjugated with a flurophor, Texas Red (TRSC, Texas red sulfonyl chloride; Jackson ImmunoResearch Laboratories, Inc., West Grove, MA). The specificity of the type $\mathrm{X}$ collagen $\mathrm{NC1}$ antibody for immunohistochemistry studies has been demonstrated previously (16).

Samples for ultrastructural analysis were prepared as described previously (24). In brief, a slice of the core of metaphyseal biopsy was cut into longitudinal blocks for orientation and fixation in Karnovsky's fixative. After $2 \mathrm{~h}$ the blocks were rinsed in PBS and stored before postfixation in osmium tetroxide. They were then dehydrated in graded acetone and embedded in Spurr low-viscosity embedding resin (ProSciTech, Thuringowa, Qld., Australia). Ultrathin sections were cut using a Reichert Ultramicrotome (C. Reichert AG, Vienna, Austria), and were stained with uranyl acetate and lead citrate (24). Stained sections were viewed with a CM12 electron microscope (Philips Scientific Pty. Ltd., Sydney, Australia) and micrographs were prepared.

\section{Results}

Characterization of the mutation. Overlapping PCR fragments spanning the entire COL10A1 gene coding sequence were analyzed for mutations by the chemical cleavage method (19). The only PCR product that contained a mismatch was the 745bp fragment covering bases 1491-2235 of the cDNA. After modification with osmium tetroxide, unique cleavage products with piperidine were identified, indicating that there was an allelic sequence variation within the $\mathrm{NC1}$ domain with mismatched $\mathrm{C}$ and $\mathrm{T}$ within the heteroduplexes (Fig. $2 a$ ). To identify the base change, a shorter 510-bp PCR fragment containing the mismatch was cloned and sequenced. Sequencing revealed that in 6 out of the $10 \mathrm{cDNA}$ clones sequenced, there was a base change of $\mathrm{C}^{1992}$ to $\mathrm{A}$ that changed the codon TAC for Try ${ }^{632}$ to a stop codon TAA (Fig. $2 b$ ), predicting synthesis of a truncated type $\mathrm{X}$ collagen chain shortened by 48 amino acids. The mutation also introduced an additional BsrI restriction enzyme cleavage site in the DNA sequence and this was used to confirm the base change on multiple PCR products amplified from the patient (see below). The origin of this mutation was not determined, as DNA samples were not available from the parents.

In vitro expression and assembly of normal and mutant $\alpha 1(X)$. The mutation $\mathrm{Y} 632 \mathrm{X}$ was constructed in a full-length human type $X$ collagen cDNA plasmid, pTM1-h10wt (3). As expected, in vitro-coupled transcription and cell-free translation produced a truncated $\alpha 1(\mathrm{X})$ chain (Fig. 3, lane 2) with an apparent molecular weight consistent with the predicted truncation of 48 amino acids at the carboxyl terminus when compared with the normal $\alpha 1$ (X) (Fig. 3, lane 1). While the normal $\alpha 1(\mathrm{X})$ chains assembled into trimers during the in vitro trans- 
(a)

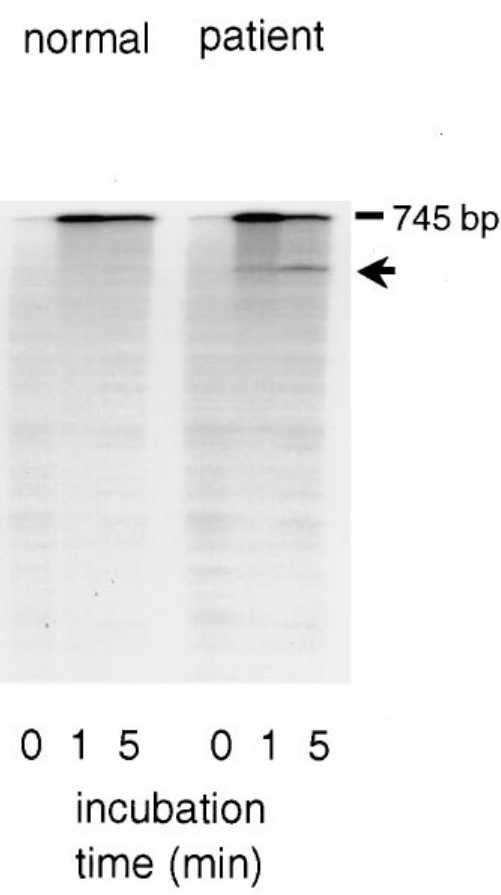

(b)

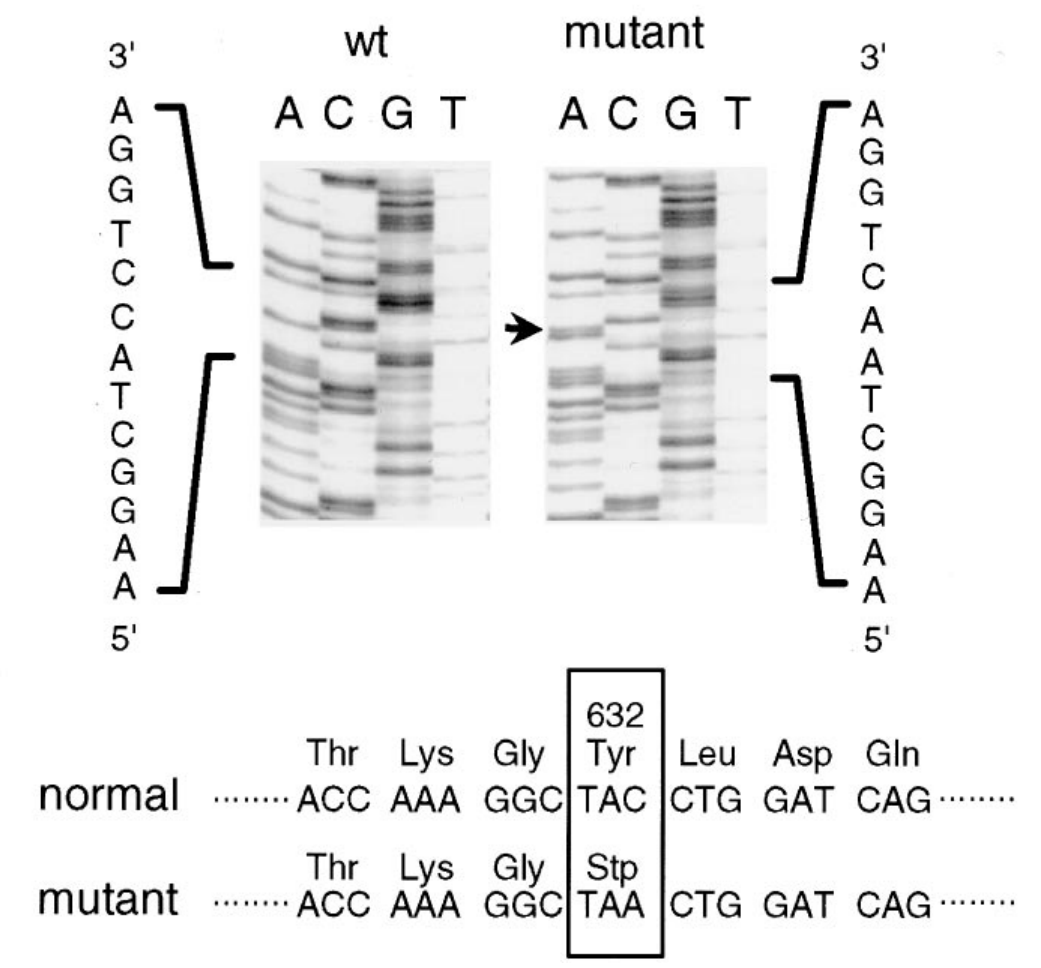

Figure 2. Chemical cleavage analysis of PCR-generated heteroduplexes and DNA sequence analysis of normal and mutant clones. (a) A 745 bpfragment covering the most $3^{\prime}$ region of the COL10A1 gene was amplified using the PCR under conditions favoring heteroduplex formation (see Methods for details). The products were end-labeled at the $5^{\prime}$ end with $\left[\gamma^{-}{ }^{32} \mathrm{P}\right] \mathrm{ATP}$ modified with osmium tetroxide for 0,1 , and 5 min as indicated, cleaved with piperidine, and the resultant fragments resolved on a $5 \%$ denaturing polyacrylamide gel. A unique cleavage fragment was identified in the patient's sample (arrow). (b) The PCR fragment containing the mismatch was cloned into the pBluescript II KS (+) and sequenced (see Methods for details). A single C-A base mutation converting the codon TAC for Tyr ${ }^{632}$ to a termination codon TAA is shown (boxed), and the arrow identifies the abnormal base in the mutant clone sequence.

lation, the mutant $\alpha 1(\mathrm{X})$ chains were present only as monomers (Fig 3, lane 2).

Analysis of type $X$ collagen expression in growth plate cartilage. Type $\mathrm{X}$ collagen was extracted from growth plate cartilage and analyzed by immunoblotting with a polyclonal antibody to the triple-helical (COL1) domain that can detect both the normal and mutant truncated $\alpha 1(\mathrm{X})$ chains produced in vitro (Fig. 3, lanes 3 and 4). Such an analysis of cartilage extracts detected only normal-sized type $\mathrm{X}$ collagen chains with no indication of the truncated mutant chains (Fig. 3, lane 6). Furthermore, immunoprecipitation of $\left[{ }^{35} \mathrm{~S}\right]$ methionine-labeled type $\mathrm{X}$ collagen from the patient's growth plate cartilage showed normal chains that were assembled into trimers with no detectable truncated mutant chains (Fig. 3, lane 10). The ability of the NC1 antibody to immunoprecipitate both wild-type and mutant type $\mathrm{X}$ collagen chains is demonstrated in Fig. 3, lanes 7 and 8 . The absence of truncated mutant protein could be due to protein degradation or to reduced steady-state mRNA levels, which commonly occurs in mRNAs containing premature termination mutations $(25,26)$.

To determine the relative levels of mutant and normal $\alpha 1(\mathrm{X})$ mRNA, two sensitive molecular techniques were used. First, since the mutation introduced an additional Bsr I restriction enzyme site, RT-PCR of total RNA extracted from growth plate cartilage followed by Bsr I digestion was used to determine if mutant mRNA was present. Because a single exon (exon 3) codes for the majority of the protein sequence, it was critical to ensure that the PCR products were amplified specifically from the mRNA rather than from potential contaminating genomic DNA. This goal was achieved by a twostage amplification where first the entire $2.1 \mathrm{~kb}$ of coding sequence (exons 2 and 3) was amplified. PCR products from genomic DNA would be much larger because of intron 2 sequence amplification. The second-stage PCR amplified a shorter 510-bp fragment (bases 1726-2235) using primers BX1 and HX6 (Fig. 4) containing the mutation from this 2.1-kb template. The Bsr I restriction digest of this cDNA-derived fragment and similar fragments amplified from patient and control genomic DNA are shown in Fig. 4. Amplification of patient genomic DNA demonstrated the presence of the mutant allele 244-bp and 140-bp Bsr I restriction fragments (lane 3) along with the normal allele 384-bp and 126 bands (Fig. 4, lane 2). In contrast, RT-PCR of the mRNA from the patient (Fig. 4, lane 6) produced an identical pattern to the control (Fig. 4, lane 6) with no evidence of the mutant-specific 244-bp and 140-bp fragments.

The second approach to detect and quantify mutant mRNA was carried out using allele-specific single nucleotide primer extension cDNA products amplified from growth cartilage mRNA by RT-PCR. The 18-mer oligonucleotide used for 
(a)

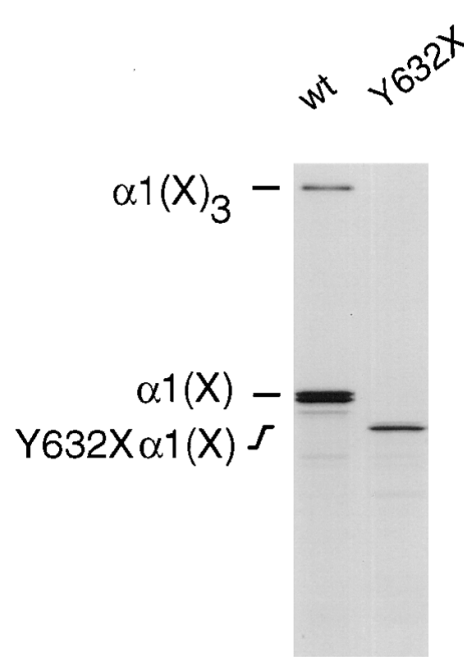

(b)
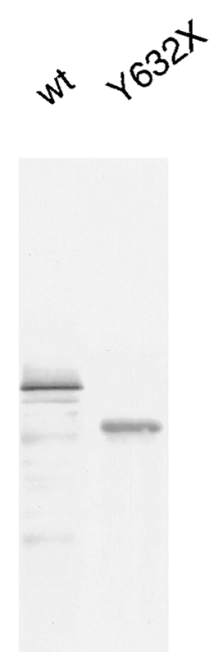

34
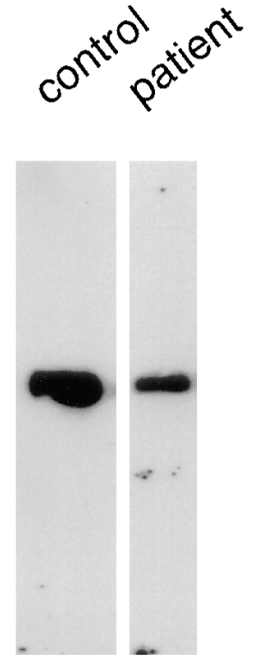

56 (c)

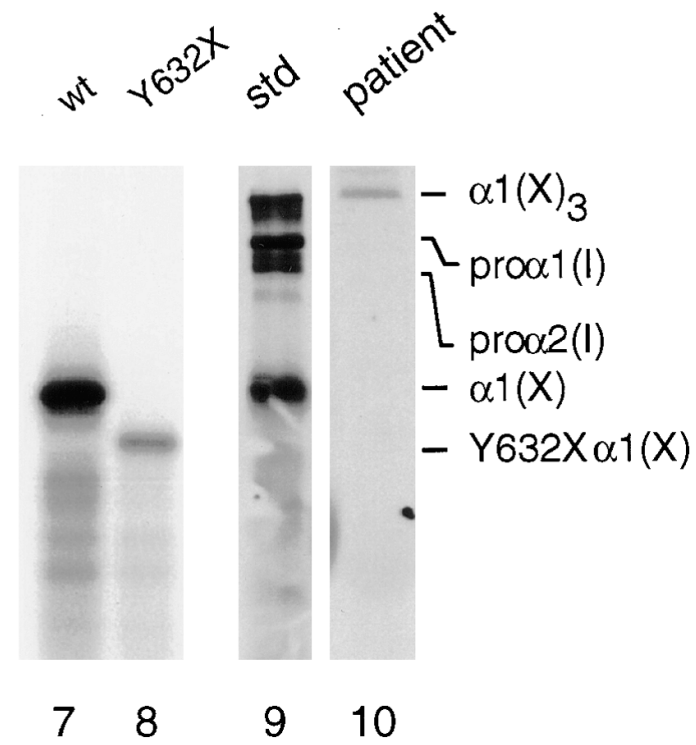

Figure 3. In vitro and in vivo analysis of type X collagen. (a) Normal ( $w t)$ and mutant ( $Y 632 X)$ type X collagen cDNA constructs were transcribed and translated in a coupled cell-free translation system in the presence of canine microsomal membranes labeled with $\left[{ }^{35} S\right]$ methionine. (b) Immunoblot analysis of wt and mutant type X collagens from cell-free translation (lanes 3 and 4), and protein extracts from a control and the patient's growth plate cartilage (lanes 5 and 6 ). Proteins were resolved by SDS/PAGE and transferred onto a nylon membrane followed by hybridization to a polyclonal antibody specific for type X collagen and detected by chemiluminescence. (c) Type X collagen antibody immunoprecipitate of wt (lane 7) and mutant (lane 8) cell-free translation products. Type X collagen produced by growth plate cartilage from the patient was also biosynthetically labeled with $\left[{ }^{35} \mathrm{~S}\right]$ methionine and immunoprecipitated (lane 10). Lane 9 is a $\left[{ }^{3} \mathrm{H}\right]$ proline-labeled medium fraction from UMR106-01 cells transfected with the normal type $\mathrm{X}$ collagen cDNA construct (14). This sample was partially denatured at $100^{\circ} \mathrm{C}$ for 5 min to establish the migrational position of the monomer and trimer forms of normal type X collagen. All other samples were analyzed on a $7.5 \%$ gel under reducing conditions, and were denatured at $60^{\circ} \mathrm{C}$ for $10 \mathrm{~min}$ before electrophoresis. The migration positions of wt monomer $[\alpha 1(\mathrm{X})]$ and trimer $\left[\alpha 1(\mathrm{X})_{3}\right]$, and the truncated mutant monomer [Y632X $\left.\alpha 1(\mathrm{X})\right]$ forms are indicated. Migration of the endogeneous UMR106-01 type I collagen pro- $\alpha 1(\mathrm{I})$ and pro- $\alpha 2(\mathrm{I})$ chains are also shown. Details of the experimental procedures are described in the Methods section.

primer extension analysis annealed $5^{\prime}$ and one base short of the mutation (Fig. 5). Extension with radioactive dCTP will detect primer extension of two cytosines from template sequence originating from the normal allele resulting in a labeled 20-mer. The use of radioactive dATP will detect specific primer extension by one adenosine base from the mutant allele templates resulting in a labeled 19-mer. This analysis showed the expected extension from templates amplified from
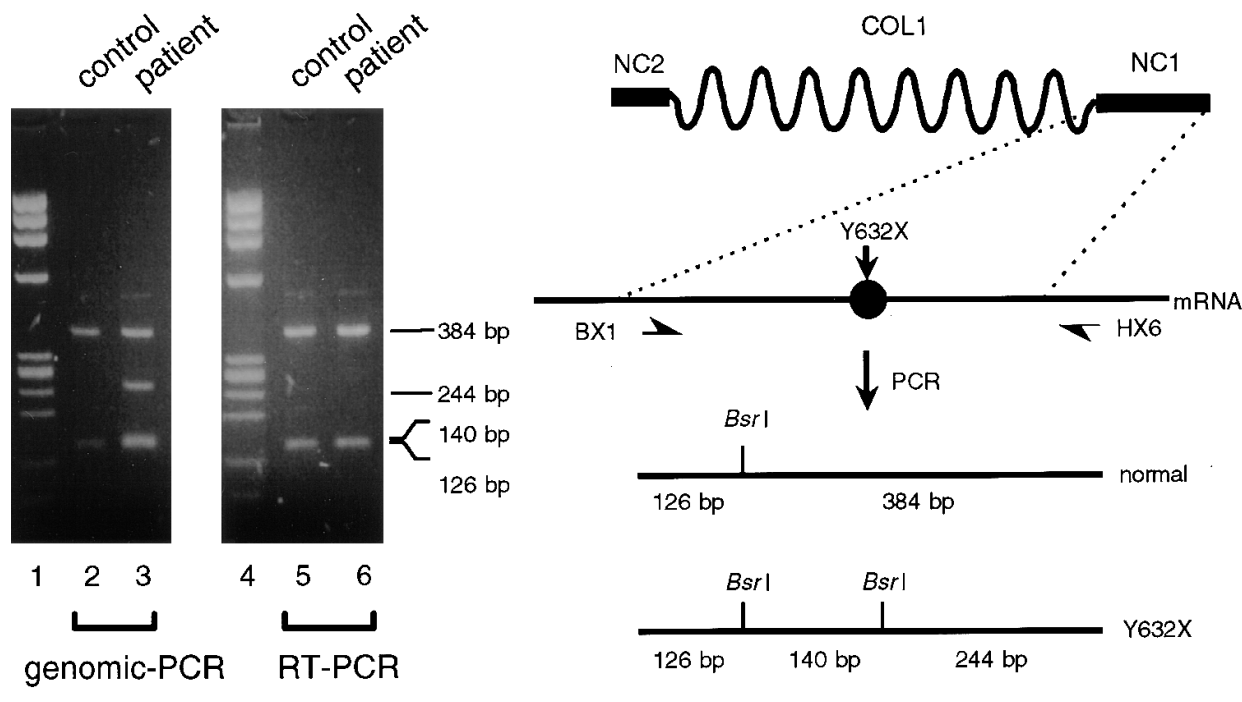

Figure 4. Bsr I restriction mapping of PCR-amplified fragments from genomic DNA and by RT-PCR from mRNA. A 510-bp fragment covering bp 1726-2235 of the type X collagen cDNA sequence was amplified from genomic DNA (lanes 2 and 3) and mRNA (lanes 5 and 6 ) prepared from a control and the patient as described in Methods. Restriction mapping was used to identify the mutant and normal sequences as the mutation introduced an addition Bsr I site and new 244-and 140-bp restriction fragments would be expected. The identities of the various lanes are indicated. Lanes 1 and 4 are Hae III-digested $\phi$ X174 DNA molecular weight markers. The Bsr I restriction maps and the size of the fragments from the normal and mutant PCR products are shown next to the figure. The position and orientation of the primers (BX1 and HX6) are shown by arrows. 


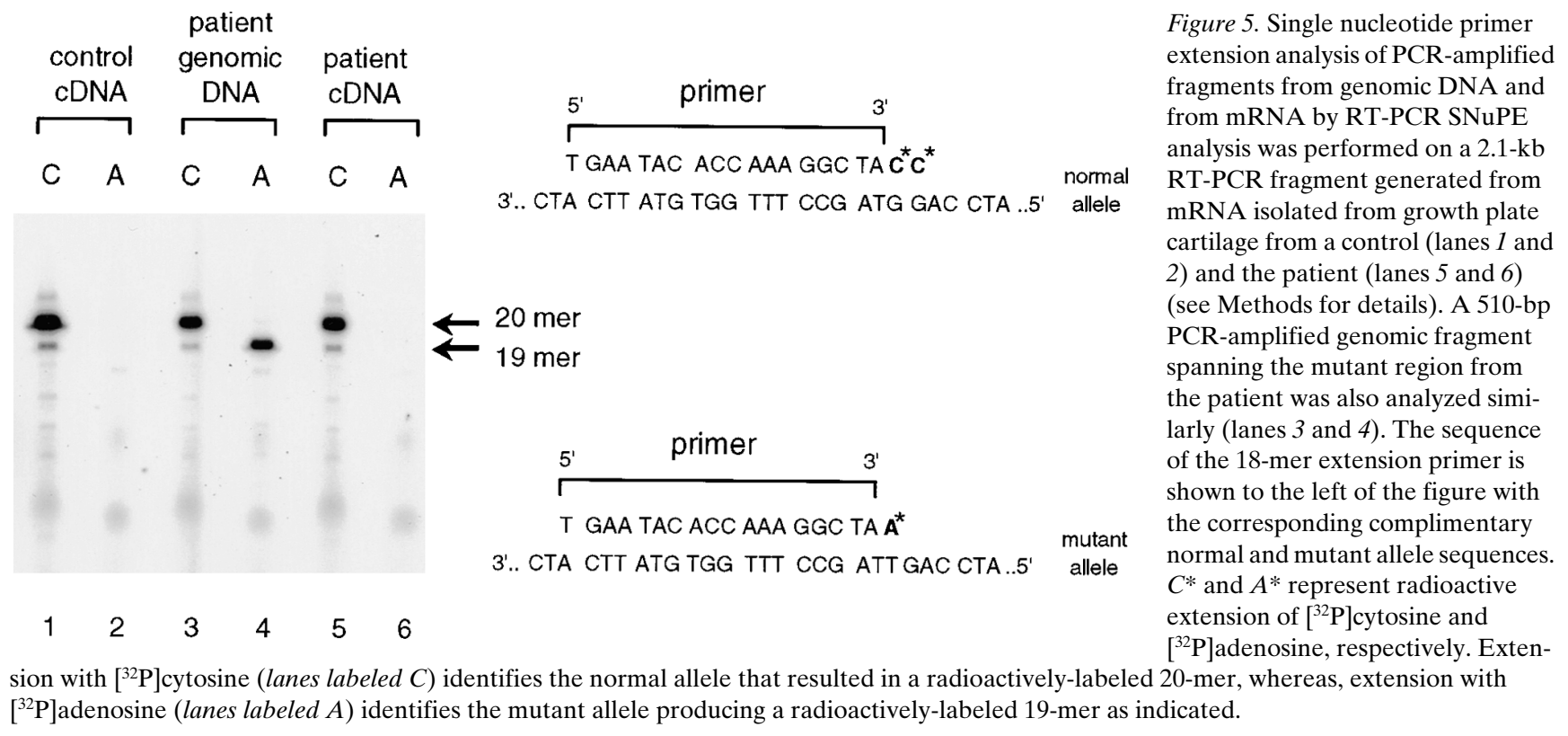

the patient's genomic DNA, with extension from both the normal and mutant alleles (Fig. 5, lanes 3 and 4). In contrast, only the 20-mer-dCTP primer extension product of the normal allele was detected from RT-PCR templates amplified from the patient's mRNA (Fig. 5, lanes 5 and 6).

Immunohistochemical and ultrastructural analysis of the growth plate cartilage. Immunohistochemical analysis of the patient's growth plate cartilage demonstrated a type X collagen-containing matrix. Interestingly, while type $X$ collagen can be found distributed throughout the growth plate, localization within the pericellular matrix of hypertrophic chondrocytes is evident (Fig. 6). Histological analysis also identified areas of fibrocartilage with birefringence characteristic of type I collagen (data not shown). These areas stained positive to a type I collagen-specific antibody (Fig. 6). The growth plate cartilage was also examined by electron microscopy (Fig. 7). The cells show chondrocyte morphology and a pericellular matrix, and the fibrous matrix shows dense collagenous fibrils. There is transition to calcified fibrocartilage without the orderly transition through a hypertrophic zone. Clusters of matrix vesicles are scattered through the growth plate, but appear to be dissociated from the calcification front.

\section{Discussion}

The SMCD phenotype in the proband was shown to be due to a heterozygous point mutation in the COL10A1 gene. A transition of C1992 to A resulted in conversion of Tyr at position 632 (within the carboxyl-terminal $\mathrm{NC1}$ domain) to a stop codon, predicting synthesis of an $\alpha 1(\mathrm{X})$ truncated by 48 amino acids. While similar $\alpha 1(\mathrm{X})$ premature termination mutations have been defined in SMCD by genomic sequence analysis (4), the case reported here is the first to be followed up by detailed study of the molecular consequences of the mutation by tissue type $\mathrm{X}$ collagen analysis and in vitro expression and assembly studies. The importance of these data in understanding the molecular pathology of SMCD is highlighted by the controversies raised by the apparent inconsistencies between SMCD and $\alpha 1(\mathrm{X})$ transgenic (17) and knockout $(15,16)$ mouse models.

In summary, Colloal knockout studies produced mice that, when homozygous for the null mutation, produced a mild phenotype with some of the features of SMCD, including growth plate compression, disorganization, and coxa vara in some older mice. When heterozygous for the null mutation, the mice were phenotypically normal (16). In contrast, transgenic mice expressing a chick $\alpha 1(\mathrm{X})$ with a helix deletion displayed a spondylometaphyseal dysplasia phenotype more severe than SMCD (17). These data thus raise serious questions about whether haploinsufficiency alone could account for the pathology of human SMCD, or whether the $\alpha 1(\mathrm{X})$ mutations exerted a dominant-negative effect in vivo. Given the obvious anatomical differences and consequent growth plate weight-bearing characteristics of mouse compared with human joints, which may lead to subtly different pathological mechanisms in the two species, or indeed may indicate different structural or developmental roles for type $\mathrm{X}$ collagen, the only approach likely to resolve the issue is to undertake direct analysis of type $\mathrm{X}$ collagen expression in the growth plate cartilage of SMCD patients.

Such direct tissue analysis revealed that the mutant truncated $\alpha 1(\mathrm{X})$ was not detectable in growth plate cartilage extracts. The mild dissociative extraction procedure would not have been expected to extract type X collagen completely, but it should however provide a representative sampling of the total type $X$ collagen content. Indeed, since structurally mutant collagens are likely to be at least partially excluded from the mature organized matrix $(27,28)$, the mutant type $\mathrm{X}$ should be enriched in this readily extracted fraction. The total absence of the mutant $\alpha 1(\mathrm{X}) \mathrm{Y} 632 \mathrm{X}$ protein in the patient's growth plate cartilage predicted that the type $\mathrm{X}$ collagen content of the cartilage would be reduced to $\sim 50 \%$ of normal levels. Unfortunately this important comparative quantitative analysis was not possible because of the impossibility of obtaining age- and site-matched control growth plate cartilage.

Despite the fact that we used sensitive PCR-based tech- 

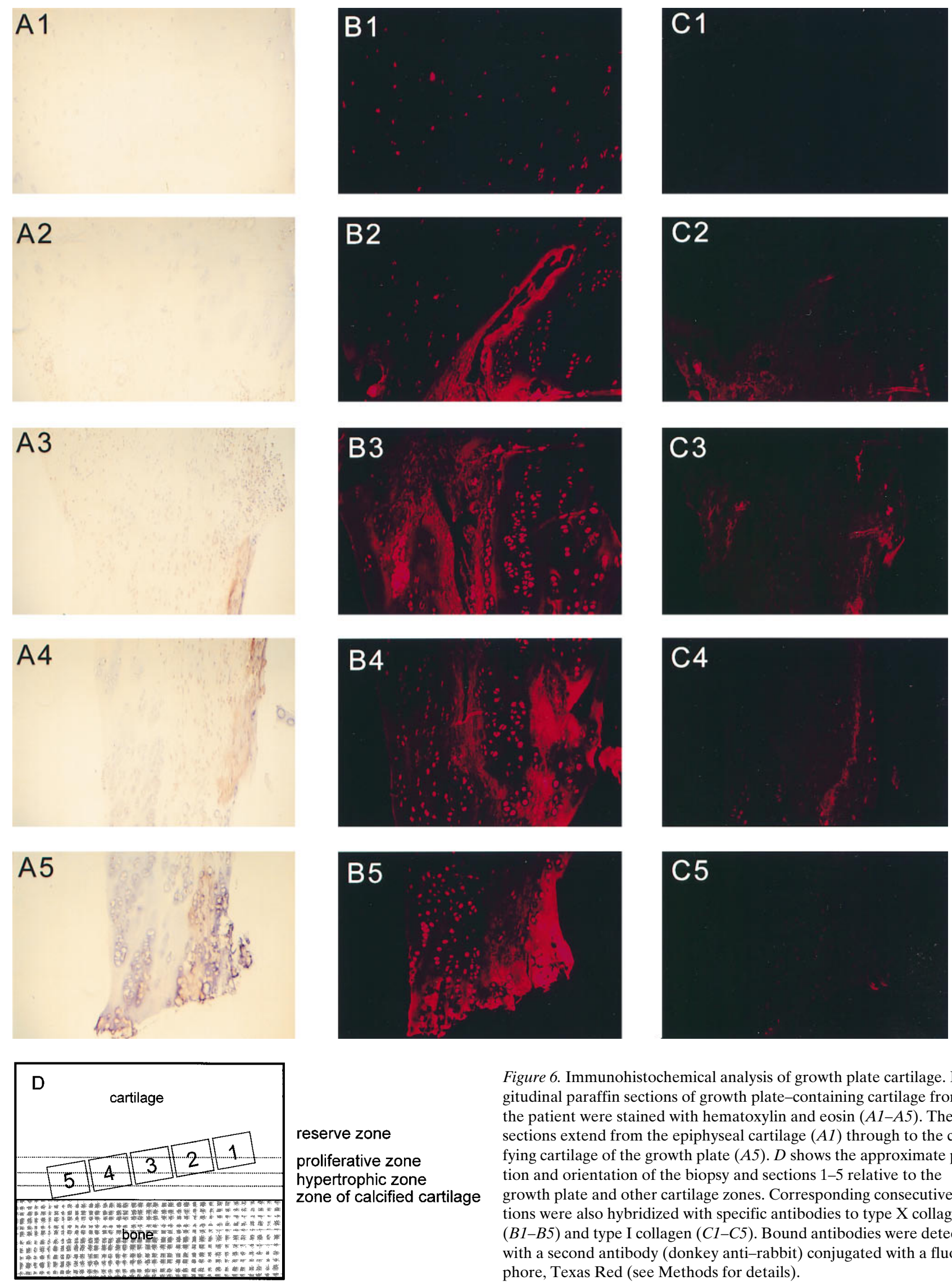

Figure 6. Immunohistochemical analysis of growth plate cartilage. Longitudinal paraffin sections of growth plate-containing cartilage from the patient were stained with hematoxylin and eosin $(A 1-A 5)$. These

reserve zone

proliferative zone hypertrophic zone zone of calcified cartilage sections extend from the epiphyseal cartilage $(A 1)$ through to the calcifying cartilage of the growth plate $(A 5)$. $D$ shows the approximate position and orientation of the biopsy and sections $1-5$ relative to the growth plate and other cartilage zones. Corresponding consecutive sections were also hybridized with specific antibodies to type $\mathrm{X}$ collagen $(B 1-B 5)$ and type I collagen $(C 1-C 5)$. Bound antibodies were detected with a second antibody (donkey anti-rabbit) conjugated with a fluorophore, Texas Red (see Methods for details). 

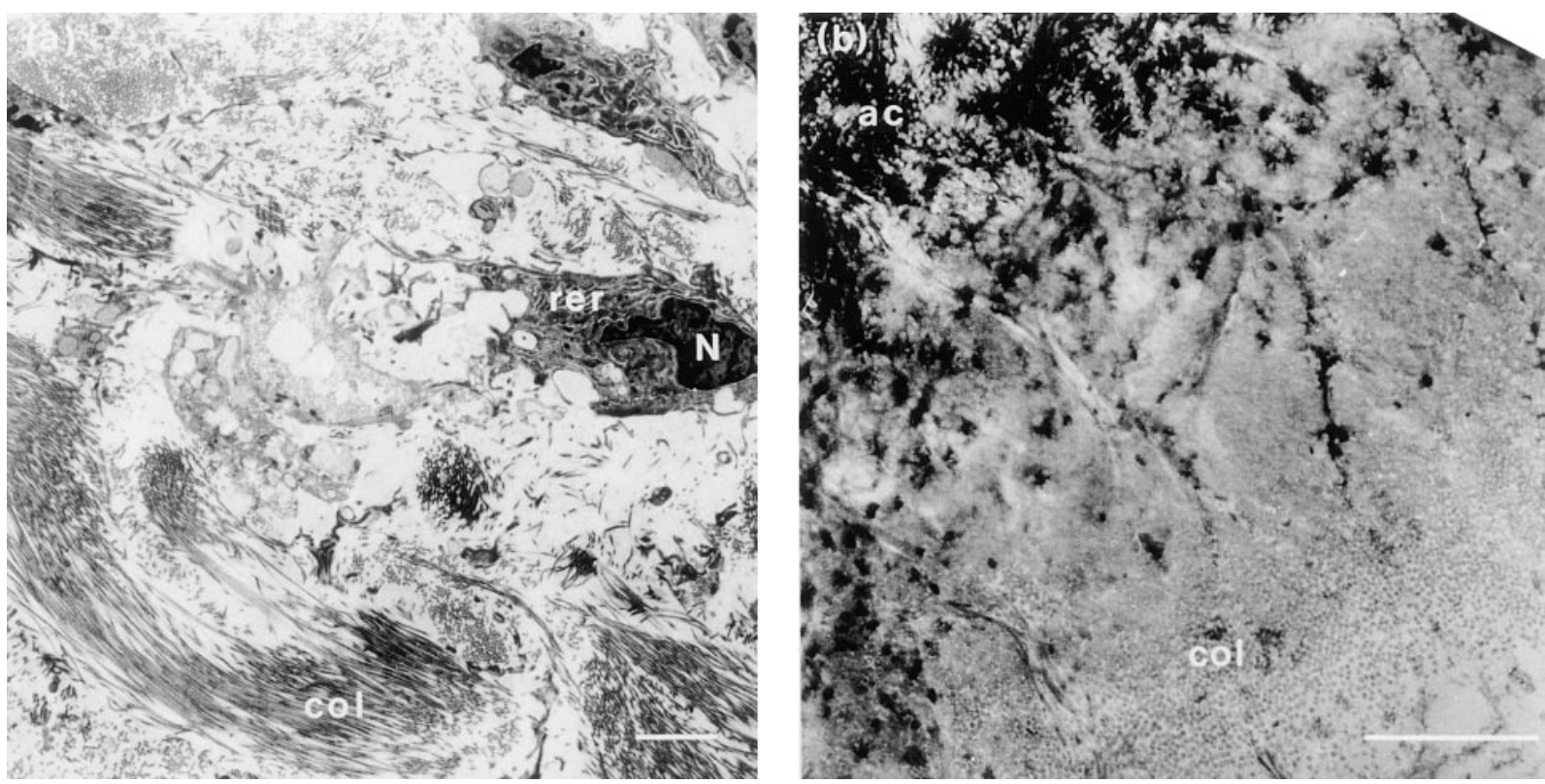

Figure 7. Ultrastructural analysis of growth plate cartilage. (a) Showed chondrocyte cluster with surrounding collagenous matrix (col). The cell nucleus $(N)$ and rough endoplasmic reticulum $(r e r)$ are indicated. The territorial (perichondrocytic) matrix is noncollagenous, and is stained with urenyl acetate and lead citrate. (b) Showed dense collagenous matrix (cross-section) with arrays of apatite crystals (ac) apparently arising from dense collagenous matrix. There are no matrix vesicles associated with the calcification front stained with uranyl acetate and lead citrate.

Bars, $2 \mu \mathrm{M}$.

niques, we were unable to detect any mutant $\alpha 1(\mathrm{X})$ mRNA in the tissue, demonstrating that the matrix deficiency of type $\mathrm{X}$ collagen was not due to instability and degradation of the truncated protein (which was unable to assemble into stable trimers), but to the absence of expression of stable mRNA from the mutant allele. These data convincingly demonstrate that in this SMCD patient, the mutant $\alpha 1(\mathrm{X})$ allele is functionally null, and that the pathology results from type $\mathrm{X}$ collagen haploinsufficiency.

Degradation of mRNAs containing premature termination mutations is a common finding in many diseases $(25,29-32)$, and this is the most likely explanation of the absence of mutant $\alpha 1(\mathrm{X})$ mRNA in the SMCD patient. The mechanism of mutant mRNA degradation is not yet fully understood, but several models involving mutation scanning during splicing and nuclear-cytoplasmic transport have been suggested, and have been recently reviewed (26). Premature termination mutations in all exons up to the final most $3^{\prime}$ exon of the gene generally target the mutant mRNA for degradation $(26,31,32)$. It is of interest to note that in the case of this SMCD patient, the mutation is within the last exon (exon 3), which would normally predict mRNA stability and synthesis of truncated protein. In the case of the COL1OA1 gene where the majority of the protein is coded by the final exon, mRNA degradation falls outside the mechanisms in the proposed models, suggesting that rather than a general single degradation mechanism that strictly adheres to proposed rules of the exonic location of the mutation, there may be either some gene specificity in how this is applied, or else other mechanisms must be involved. Thus, based on the data presented in this study, we would predict that in the other cases of SMCD resulting from nonsense mutations, the mutant mRNA would be subjected to similar degradation leading to $\alpha 1(\mathrm{X})$ haploinsufficiency.
Missense mutations of COL10A1 also cause SMCD (4), and to date no studies have been performed to determine if these mutations also result in haploinsufficiency of $\alpha 1(\mathrm{X})$ by preventing assembly and targeting of the mutant for breakdown, or whether the mutant can associate with the normal allele product and disturb assembly and function in a dominant-negative fashion. We have addressed this by site-directed mutagenesis of $\alpha 1(\mathrm{X})$ and expression of SMCD mutations in an in vitro system to study assembly in transfected cells $(3,14)$. In these studies, SMCD mutations of the NC1 domain prevented in vitro assembly of the mutant chains into trimers. In cotranslation experiments with normal $\alpha 1(\mathrm{X}), \mathrm{NC1}$ amino acid substitutions severely compromised heterotrimer assembly (14), although a minute amount of mutant/normal heterotrimer assembly in vitro was detected. However, whether or not this mutant/normal assembly can occur in transfected cells (and thus in vivo where assembly mechanisms are more complex, and stringent quality control processes occur; 33, 34), has not yet been tested.

These data are not yet conclusive, but suggest the presence of subtle biochemical heterogeneities in $\alpha 1(\mathrm{X}) \mathrm{NC1}$ missense mutations. The NC1 region of type $\mathrm{X}$ collagen contains a sequence domain of $\sim 130$ amino acid residues, including a conserved cluster of aromatic acids that has a hydrophilicity profile of marked similarity to that of the carboxyl terminal propeptides of the fibrillar collagens (35). Mutations in the $\mathrm{NC} 1$ domain that disturb this aromatic cluster or change NC1 protein folding such that hydrophobic surface interactions are impaired, would be expected to result in the observed failure to trimerize. The SMCD mutations all fall within regions of high homology (Fig. 8), thus suggesting the disrupted regions are absolutely critical for chain interactions. So far, no mutation has been defined within the $\mathrm{N}$-terminal region of the $\mathrm{NC} 1$ 


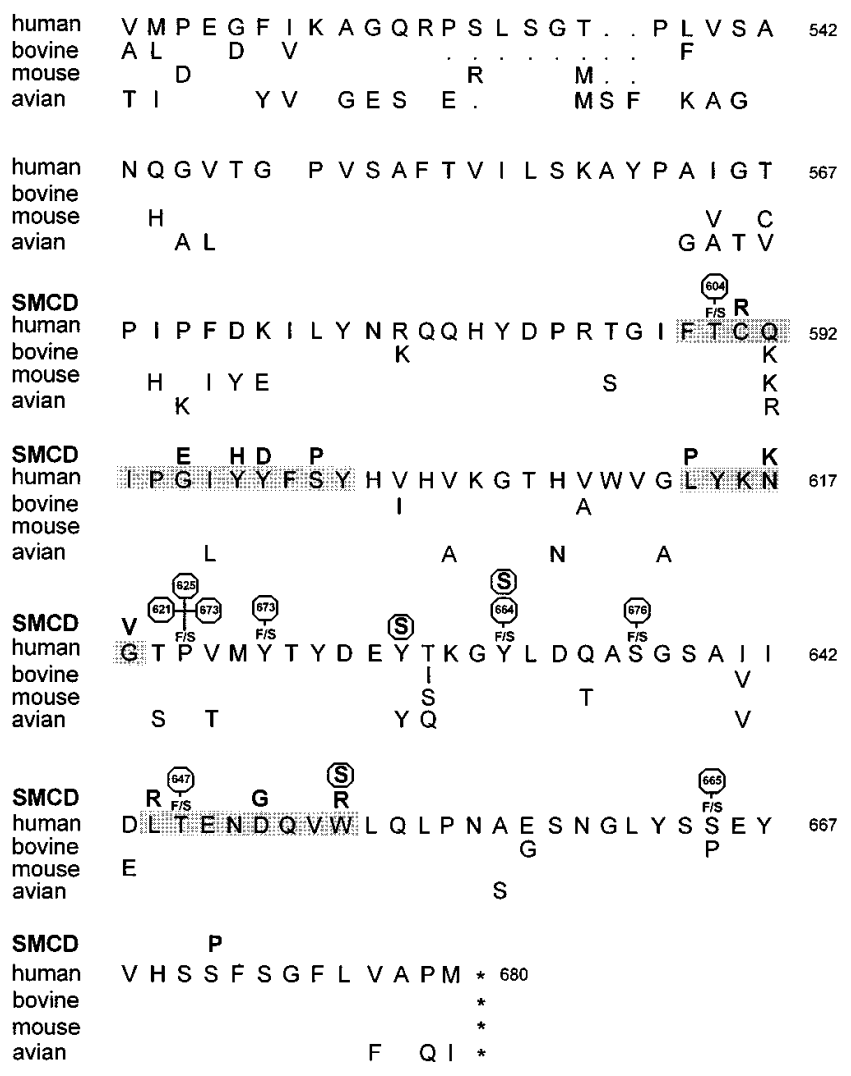

Figure 8. Comparative analysis of NC1 mutations causing SMCD.

The amino acid sequence of the human type $\mathrm{X}$ collagen $\mathrm{NC} 1$ domain is listed. Amino acid deviations between species such as bovine, mouse, and avian are also indicated with the changes listed below the corresponding human amino acid. The locations and identities of the known SMCD missense mutations are shown in bold above the corresponding normal amino acid. $S$ enclosed in an octagon represents termination mutations at the amino acids indicated, whereas $F / S$ represents frameshift mutations, and the number within the octagon designates the position of premature terminations further along the polypeptide chain. The 13-amino acid aromatic zipper domain (amino acid 589-601; 37) and two other domains (amino acid 614-618 and 644-652) with localized amino acid substitutions are shaded.

domain, which encompasses a variable (amino acid 520-546) and a highly conserved sequence (amino acid 547-562). The missense mutations appear to be localized to three subdomains: amino acids 589-601, amino acids 614-618, and amino acids 644-652. Mutations in the first subdomain are consistent with disruption of the 13-amino acid aromatic zipper motif proposed to be important in chain association (35).

In this patient, cartilage from the femoral neck showed a collagenous fibrocartilage by electron microscope consistent with either a relative decrease in cartilage proteoglycans or an induction of type I collagen synthesis. In a type X collagen knock out mouse (16), there was a similar decrease in proteoglycan with the hypertrophic zone. This patient also showed dissociation between the localization of matrix vesicles and the calcification front, similar to the finding in the type $\mathrm{X}$ collagen knock out mouse (16). The areas of fibrocartilage in the growth plate cartilage most likely result from microfracture repair. The presence of microfractures in the growth plate have been reported in the transgenic mouse expressing a shortened $\alpha 1(\mathrm{X})$ chain (17). In this mouse model and in the SMCD patient, the microfractures most probably result from the structural inadequacies of the growth plate due to the type $\mathrm{X}$-deficient matrix during weight bearing.

\section{Acknowledgments}

This work was supported by grants from the National Health and Medical Research Council of Australia and the Royal Children's Hospital Research Foundation.

\section{References}

1. Lachman, R.S., D.L. Rimoin, and J. Spranger. 1988. Metaphyseal chondrodysplasia, Schmid type. Clinical and radiographic delineation with a review of the literature. Pediatr. Radiol. 18:93-102.

2. Warman, M.L., M. Abbott, S.S. Apte, T. Hefferon, I. McIntosh, D.H. Cohn, J.T. Hecht, B.R. Olsen, and C.A. Francomano. 1993. A type X collagen mutation causes Schmid metaphyseal chondrodysplasia. Nat. Genet. 5:79-82.

3. Chan, D., W.G. Cole, J.G. Rogers, and J.F. Bateman. 1995. Type X collagen multimer assembly in vitro is prevented by a Gly618 to Val mutation in the $\alpha 1(\mathrm{X}) \mathrm{NC} 1$ domain resulting in Schmid metaphyseal chondrodysplasia. $J$. Biol. Chem. 270:4558-4562.

4. Wallis, G.A., B. Rash, B. Sykes, J. Bonaventure, P. Maroteaux, B. Zabel, R. Wynne-Davies, M.E. Grant, and R.P. Boot-Handford. 1996. Mutations within the gene encoding the $\alpha 1$ (X) chain of type X collagen (COL10A1) cause metaphyseal chondrodysplasia type Schmid but not several other forms of metaphyseal chondrodysplasia. J. Med. Genet. 33:450-457.

5. Apte, S.S. and B.R. Olsen. 1993. Characterization of the mouse type X collagen gene. Matrix. 13:165-179.

6. Schmid, T.M. and T.F. Linsenmayer. 1983. A short-chain (pro)collagen from aged endochondral chondrocytes. Biochemical characterization. J. Biol. Chem. 258:9504-9509.

7. Kielty, C.M., A.P. Kwan, D.F. Holmes, S.L. Schor, and M.E. Grant. 1985 Type X collagen, a product of hypertrophic chondrocytes. Biochem. J. 227:545-554.

8. Nerlich, A.G., T. Kirsch, I. Wiest, P. Betz, and K. von der Mark. 1992. Localization of collagen $\mathrm{X}$ in human fetal and juvenile articular cartilage and bone. Histochemistry. 98:275-281.

9. Grant, W.T., G.J. Wang, and G. Balian. 1987. Type X collagen synthesis during endochondral ossification in fracture repair. J. Biol. Chem. 262:98449849.

10. Reichenberger, E., T. Aigner, K. von der Mark, H. Stoss, and W. Bertling. 1991. In situ hybridization studies on the expression of type X collagen in fetal human cartilage. Dev. Biol. 148:562-572.

11. Thomas, J.T., C.J. Cresswell, B. Rash, H. Nicolai, T. Jones, E. Solomon, M.E. Grant, and R.P. Boot-Handford. 1991. The human collagen X gene. Complete primary translated sequence and chromosomal localization. Biochem. J. 280:617-623.

12. LuValle, P., Y. Ninomiya, N.D. Rosenblum, and B.R. Olsen. 1988. The type $\mathrm{X}$ collagen gene. Intron sequences split the 5 '-untranslated region and separate the coding regions for the non-collagenous amino-terminal and triplehelical domains. J. Biol. Chem. 263:18378-18385.

13. Reichenberger, E., F. Beier, P. LuValle, B.R. Olsen, K. von der Mark, and W.M. Bertling. 1992. Genomic organization and full-length cDNA sequence of human collagen X. FEBS Lett. 311:305-310.

14. Chan, D., Y.M. Weng, A.M. Hocking, S. Golub, D.J. McQuillan, and J.F. Bateman. 1996. Site-directed mutagenesis of human type X collagen. Expression of $\alpha 1(\mathrm{X}) \mathrm{NC} 1, \mathrm{NC} 2$, and helical mutations in vitro and in transfected cells. J. Biol. Chem. 271:13566-13572.

15. Rosati, R., G.S. Horan, G.J. Pinero, S. Garofalo, D.R. Keene, W.A. Horton, E. Vuorio, B. de Crombrugghe, and R.R. Behringer. 1994. Normal long bone growth and development in type X collagen-null mice. Nat. Genet. 8: $129-135$.

16. Kwan, K.M., M.K. Pang, S. Zhou, S.K. Cowan, R.Y. Kong, T. Pfordte, B.R. Olsen, D.O. Sillence, P.P. Tam, and K.S. Cheah. 1997. Abnormal compartmentalization of cartilage matrix components in mice lacking collagen X: implications for function. J. Cell Biol. 136:459-471.

17. Jacenko, O., P.A. LuValle, and B.R. Olsen. 1993. Spondylometaphyseal dysplasia in mice carrying a dominant-negative mutation in a matrix protein specific for cartilage-to-bone transition. Nature. 365:56-61.

18. Douglas, A.M., A.M. Georgalis, L.R. Benton, K.L. Canavan, and B.A. Atchison. 1992. Purification of human leucocyte DNA: proteinase K is not necessary. Anal. Biochem. 201:362-365.

19. Cotton, R.G.H., N.R. Rodrigues, and R.D. Campbell. 1988. Reactivity of cytosine and thymine in single base pair mismatches with hydroxylamine and osmium tetroxide and its application to the study of mutations. Proc. Natl. Acad. Sci. USA. 85:4397-4401.

20. Gough, N.M. 1988. Rapid and quantitative preparation of cytoplasmic 
RNA from small numbers of cells. Anal. Biochem. 173:93-95.

21. Gibson, G.J., K.T. Francki, J.J. Hopwood, and B.K. Foster. 1991. Human and sheep growth-plate cartilage type $\mathrm{X}$ collagen synthesis and the influence of tissue storage. Biochem. J. 277:513-520.

22. Bateman, J.F., T. Mascara, D. Chan, and W.G. Cole. 1984. Abnormal type I collagen metabolism by cultured fibroblasts in lethal perinatal osteogenesis imperfecta. Biochem. J. 217:103-115.

23. Bateman, J.F., D. Chan, T. Mascara, J.G. Rogers, and W.G. Cole. 1986. Collagen defects in lethal perinatal osteogenesis imperfecta. Biochem. J. 240: 699-708

24. Sillence, D.O., W.A. Horton, and D.L. Rimoin. 1979. Morphologic studies in the skeletal dysplasia. Am. J. Pathol. 96:813-870.

25. McIntosh, I., A. Hamosh, and H.C. Dietz. 1993. Nonsense mutations and diminished mRNA levels. Nat. Genet. 4:219.

26. Maquat, L.E. 1995. When cells stop making sense: effects of nonsense codons on RNA metabolism in vertebrate cells. RNA. 1:453-465.

27. Bateman, J.F., and S.B. Golub. 1994. Deposition and selective degradation of structurally-abnormal type I collagen in a collagen matrix produced by osteogenesis imperfecta fibroblasts in vitro. Matrix Biol. 14:251-262.

28. Mundlos, S., D. Chan, Y.M. Weng, D.O. Sillence, W.G. Cole, and J.F. Bateman. 1996. Multiexon deletions in the type I collagen COL1A2 gene in osteogenesis imperfecta type IB. Molecules containing the shortened $\alpha 2(\mathrm{I})$ chains show differential incorporation into the bone and skin extracellular matrix. $J$. Biol. Chem. 271:21068-21074.

29. Willing, M.C., S.P. Deschenes, R.L. Slayton, and E.J. Roberts. 1996. Premature chain termination is a unifying mechanism for COL1A1 null alleles in Osteogenesis Imperfecta Type I cell strains. Am. J. Hum. Genet. 59:799-809.

30. Carter, M.S., J. Doskow, P. Morris, S. Li, R.P. Nhim, S. Sandsted, and M.F. Wilkinson. 1995. A regulatory mechanism that detects premature nonsense codons in T-cell receptor transcripts in vivo is reversed by protein synthesis inhibitors in vitro. J. Biol. Chem. 270:28995-29003.

31. Zhang, Z.X., N. Wakamatsu, E.H. Mules, G.H. Thomas, and R.A Gravel. 1994. Impact of premature stop codons on mRNA levels in infantile Sandhoff disease. Hum. Mol. Genet. 3:139-145.

32. Mashima, Y. A. Murakami, R.G. Weleber, N.G. Kennaway, L. Clarke, T. Shiono, and G. Inana. 1992. Nonsense-codon mutations of the ornithine aminotransferase gene with decreased levels of mutant mRNA in gyrate atrophy. Am. J. Hum. Genet. 51:81-91.

33. Bateman, J.F., S.R. Lamande, H.H. Dahl, D. Chan, T. Mascara, and W.G. Cole. 1989. A frameshift mutation results in a truncated nonfunctional carboxyl-terminal pro $\alpha 1$ (I) propeptide of type I collagen in osteogenesis imperfecta. J. Biol. Chem. 264:10960-10964.

34. Lamande, S.R., S.D. Chessler, S.B. Golub, P.H. Byers, D. Chan, W.G. Cole, D.O. Sillence, and J.F. Bateman. 1995. Endoplasmic reticulum-mediated quality control of type I collagen production by cells from osteogenesis imperfecta patients with mutations in the pro $\alpha 1$ (I) chain carboxyl-terminal propeptide which impair subunit assembly. J. Biol. Chem. 270:8642-8649.

35. Brass, A., K.E. Kadler, J.T. Thomas, M.E. Grant, and R.P. Boot-Handford. 1992. The fibrillar collagens, collagen VIII, collagen X and C1q complement proteins share a similar domain in their $\mathrm{C}$-terminal non-collagenous regions. FEBS Lett. 303:126-128. 